Pak. J. Agri., Agril. Engg., Vet. Sci., 2020, 36 (2): 129-134

ISSN: 1023-1072 (Print), ISSN: 2663-7863 (Online)

https://doi.org/10.47432/2020.36.2.6

\title{
CORRELATION ANALYSIS BETWEEN MORPHOLOGICAL, PHYSIOLOGICAL AND YIELD TRAITS UNDER SALINITY STRESS CONDITION IN WHEAT (TRITICUM AESTIVUM L.) GENOTYPES
}

\author{
N. Gandahi ${ }^{1}$, A. W. Baloch ${ }^{* 1}$, S. M. Sarki ${ }^{2}$, M. M. Lund ${ }^{3}$ and M. N. Kandhro ${ }^{3}$ \\ ${ }^{1}$ Department of Plant Breeding and Genetics, ${ }^{2}$ Department of Soil Science, \\ ${ }^{3}$ Department of Agronomy, Sindh Agriculture University, Tandojam, Pakistan
}

\begin{abstract}
Salinity is one of the key factors reducing the plant growth and productivity of major crops including wheat. However, identifying the multiple genetic parameters associated with salt tolerance during different growth stages is critical for the evaluation and enhancement of wheat genotypes. In this context, a field experiment in non-saline and saline soil was carried out for correlation analysis of 17 morphological, physiological and yield traits in 22 genotypes of wheat. The experiment was carried out at experimental field of NIA, Tandojam during 2018-2019 in a Randomized Complete Block Design (RCBD) with three replications and two treatments (non-saline soil and salinity level of $12 \mathrm{dS} \mathrm{m}^{-1}$ ). The obtained mean squares showed significant differences among the tested genotypes for all the studied characters, reflecting that there are abundant genetic variations present in these wheat genetic resources for further utilization in wheat improvement programs. The correlation analysis demonstrated that a variety of characters, including spike length (non-saline $r=0.59^{* *}$; salinity $r=0.37^{*}$ ), spikelets spike $^{-1}$ (non-saline $r=0.49^{* *}$; salinity $r=0.46^{* *}$ ), grains spike $e^{-1}$ (non-saline $r=0.49^{* *}$; salinity $r=0.43^{* *}$ ), biological yield plot $^{-1}$ (non-saline $r=0.56^{* *}$; salinity $r=0.70^{*}$ ), harvest index (non-saline $r=0.24$; salinity $r=0.26$ ), relative water content (non-saline $r=0.53^{*}$; salinity $r=0.50^{*}$ ), seed index (nonsaline $r=0.29^{*}$; salinity $r=0.52^{* *}$ ) and $K^{+}$(non-saline $r=0.28^{*}$; salinity $r=0.32^{* *}$ ) showed positive and significant associations with grain yield plot $^{-1}$ in non-saline and salinity stress conditions. The results suggest that these above-mentioned characters may be set as selection criteria in breeding study to improve wheat genotypes for high yielding under saline filed conditions.
\end{abstract}

Keywords: correlation, salinity, wheat, yield, yield components

\section{INTRODUCTION}

Bread wheat is an annual herb, belonging to the family poaceae, which is cultivated worldwide (Belderok et al., 2000). According to an estimate, more than $60 \%$ of people in Pakistan are entirely dependent on wheat for their daily diet (Shah et al., 2017). There are multiple uses of wheat; it is staple food of masses and major ingredient of animal feed. The wheat cultivation in the country was recorded on an area of 8740 thousand hectares during 2018-19, indicating 0.6 percent decrease in area under this crop over preceding year (GoP, 2019). Soil salinity is considered as one of most important abiotic stresses, which affects adversely on germination of seeds, plant growth and productivity (Sairam et al., 2002). Decline in productivity of economic characters and even death of the crop plants is quite expected due to high extent of soil salinity (Garciadeblás et al., 2003; Qados, 2011). Many

Corresponding author: balochabdulwahid@yahoo.com crop plants have established mechanisms whether to eliminate salts from their tissues or to tolerate within the tissues (Kumar et al., 2005; Carillo et al., 2011). While some mechanisms have been developed in other crops, such as osmotic adjustment, $\mathrm{K}^{+}$retention and tissue tolerance process, but these are more difficult for bread wheat because of its large, complicated and hexaploid genome (Marcussen et al., 2014; Wang and Xia, 2018). Nevertheless, the mechanisms underlying salinity tolerance in wheat, including leaf $\mathrm{Na}^{+}$exclusion mediated by high-affinity $\mathrm{K}^{+}$transporters and reactive oxygen species detoxification have been addressed (Munns and Gilliham, 2015). Correlation analysis is used as an effective tool to determine the relationship among different traits in genetic diverse population for enhancement of crop improvement process (Dhami et al., 2018; Kandel et al., 2018). In plant breeding, the correlations are very important because of its reflection in dependence degree between two or 
more traits. Correlation analysis shows the intensity of dependence (correlation) between studied traits. Correlations disclose the extent and direction of the linking between yield and its contributing traits. Correlation studies stretch a perfect depiction of traits association which is commonly happened due to gene linkage, pleiotrophic effects, physiological association in developmental and biochemical pathway (Chitralekha et al., 2018). In wheat breeding, the breeders mostly elucidate the relationship between grain yield and other agronomic and morphological traits by using simple correlation (Kharel et al., 2018). Chitralekha et al. (2018) reported that seed yield plant ${ }^{-1}$ exhibited highly significant and positive correlations with number of seeds spike ${ }^{-1}$. Recently in Pakistan, various researchers (Baloch et al., 2014; Sohail et al., 2018; Rattar, 2019; Khan, 2020) found that traits like spike length, grains spike ${ }^{-1}$, seed index, biological yield and harvest index disclosed positive and significant associations with grain yield. However, these all above studies were carried out in non-saline soil conditions. Therefore, the current study was designed to identify best characters which may be used to develop improved bread wheat genotypes for commercial cultivation in saline soils.

\section{MATERIALS AND METHODS}

This study was aimed to estimate correlation in non-saline and saline soil $\left(12 \mathrm{dS} \mathrm{\textrm {m } ^ { - 1 }}\right)$ under natural field conditions. The salinity level of 12 dS $\mathrm{m}^{-1}$ was used since the threshold level of salinity for wheat crop is $6 \mathrm{dS} \mathrm{m}^{-1}$, indicating that above this level of salinity would create stress for wheat crop. In this regard, twenty two wheat genotypes of diverse origin were used, such as NIA-Sundar, NIA-Sarang, NIA-AS-14, NIA-AS-9, NIA-AS-10, NIA-2B, Hamal, NIA-5B, Salt-6, SRN-87, SRN-11, CT-49, NRC-1236, Bathoor08, Tatara, TJ-83, TD-1, Benazir, AS-2002, Sehar-2006, Kiran-95 and LU-26s for morphophysiological and yield traits. The characters days to $75 \%$ heading and days to $75 \%$ maturity were observed visually in the field, while plant height $(\mathrm{cm})$ tillers plant $^{-1}$, spike length $(\mathrm{cm})$, spikelets spike ${ }^{-1}$, grains spike ${ }^{-1}$, grain yield plot $^{-1}$ $(\mathrm{kg})$ biomass plot $^{-1}(\mathrm{~kg})$, harvest index (\%), seed index (1000-grain weight, g) were recorded after harvesting and flag leaf area $\left(\mathrm{cm}^{-2}\right)$ was measured when flag leaf was fully emerged. Relative water content and chlorophyll content were measured according to Bonnet et al. (2000) and Lichtenthaler (1987), respectively from fully fresh expanded leaves. $\mathrm{K}^{+}$and $\mathrm{Na}^{+}$ contents were measured in the toluene extract (Ansari and Flowers, 1986). The wheat genotypes were sown in two sets (non-saline and saline soil) in a plot size of $20 \times 15$ meter. The randomized complete block designs having three replicates were used for non-saline and saline experiments. Recommended fertilizer dose was also applied i.e. nitrogen @ 120 kg $\mathrm{ha}^{-1}$ (in two splits) in the form of ammonium sulphate $\left(\mathrm{NH}_{4}\right)_{2} \mathrm{SO}_{4}$; phosphorus @ $90 \mathrm{~kg} \mathrm{ha}^{-1}$ (full dose at the time of sowing) in the form of single superphosphate $\mathrm{Ca} \quad\left(\mathrm{H}_{2} \mathrm{PO}_{4}\right)_{2}$ and potassium @ $60 \mathrm{~kg} \mathrm{ha}^{-1}$ (full dose) in the form of potassium sulphate $\left(\mathrm{K}_{2} \mathrm{SO}_{4}\right)$. The analysis of variances and correlation coefficient was calculated with the help of computer package (Statistix Ver. 8.1).

\section{RESULTS AND DISCUSSION}

With respect to mean squares, the genotypes, treatments and genotypes $x$ treatments interaction were significantly different $(P \leq 0.05)$ for all the traits, such as days to $75 \%$ heading, days to $75 \%$ maturity, plant height, tillers plant ${ }^{-1}$, spikelets spike $e^{-1}$, grains spike ${ }^{-1}$, spike length, grain yield plot $^{-1}$, biological yield plot ${ }^{-1}$, harvest index, seed index, leaf area, relative water content, chlorophyll content, and $\mathrm{K}^{+}, \mathrm{Na}^{+}$and $\mathrm{K}^{+}$ $\mathrm{Na}^{+}$ratio (Table 1). The obtained results disclose that genotypes tend to possess genetic variation for different morpho-yield and physiological parameters; consequently, these bread wheat genotypes may be selected for further breeding experiments. Naggar et al. (2015) also reported combined analysis of variance across the four locations under different levels of salinity, which revealed that genotypes differed significantly for all studied traits at all locations and salinity levels.

In recent years, several breeders have been widely carried out correlation analysis in various field crops and unveiled the positive association of different yield contributing characters with seed yield, such as in rice (Hasan et al., 2013; Baloch et al., 2016), rapeseed (Banglian et al., 2017; Solanki et al., 2017; Rout et al., 2018), sunflower (Gorgieva et al., 2015; Baloch et al., 2016), maize (Aman et al., 2020), sorghum (Mengesha et al., 2019), millet (Chavan et al., 2019), barley (Hailu et al., 2016) and oat (Surje and De, 2014). In the present study, the correlations result in non-saline soil (Table 2) showed that a number of characters established positively significant associations with grain yield plot $^{-1}$, such as days to $75 \%$ maturity $\left(r=0.25^{*}\right)$, days to $75 \%$ heading $\left(r=0.53^{* *}\right)$, tillers plant ${ }^{-1}(r=$ 
$\left.0.63^{* *}\right)$, spike length $\left(r=0.59^{* *}\right)$, spikelets spike ${ }^{-1}$ $\left(r=0.49^{* *}\right)$, grains spike ${ }^{-1}\left(r=0.40^{* *}\right)$, seed index $\left(r=0.29^{*}\right)$, biological yield $\operatorname{plot}^{-1}\left(r=0.56^{* *}\right)$, harvest index $\left(r=0.24^{*}\right)$, relative water content $\left(r=0.53^{* *}\right)$ and $\mathrm{K}^{+}\left(\mathrm{r}=0.28^{*}\right)$. These characters may be preferred in selection program to introduce high yielding varieties. Mentioning the correlation findings at phenotypic and genotypic levels, Parihar et al. (2018) reported that seed yield disclosed its significant and positive associations with number of spikelets plant ${ }^{-1}$, effective tillers plant $^{-1}$ and 1000-seed weight. Singh et al. (2017) described that grain yield plant ${ }^{-1}$ shared positive significant correlations with different yield enhancing characters.

The obtained correlation results under salinity stress conditions (Table 3 ) displayed that a number of traits also made significantly positive associations with grain yield plot $^{-1}$, such as spike length $\left(r=0.37^{*}\right)$, spikelets spike ${ }^{-1}$ $\left(r=0.46_{* *}^{* *}\right)$, grains spike ${ }^{-1}\left(r=0.43^{* *}\right)$, seed index $\left(r=0.52^{* *}\right)$, biological yield $\operatorname{plot}^{-1} \quad\left(r=0.70^{* *}\right)$, harvest index $\left(r=0.26^{* *}\right)$, leaf area $\left(r=0.45^{* *}\right)$, relative water content $\left(r=0.50^{* *}\right)$ and $\mathrm{K}^{+}\left(\mathrm{r}=0.32^{* *}\right)$. These all yield attributing traits displayed positive correlations with grain yield plot $^{-1}$, signifying the value of these traits towards contribution in yield of salinity affected wheat field. Due to these positive correlations of different parameters with yield, referring the importance of such traits as criteria for evolving high yielding wheat cultivars for saline soils. Results are in consonance with those of Ahmad et al. (2013), where spike length, spikelets spike $^{-1}$, grains spike ${ }^{-1}$ and seed index disclosed positive and significant associations with grain yield plant $^{-1}$ under $\mathrm{NaCl}$ stress. Recently, Gadallah et al. (2017) also observed a significantly positive association of grain yield with $\mathrm{K}^{+}$and $\mathrm{K}^{+} / \mathrm{Na}^{+}$ratio under salinity stress in wheat crop. More recently, Waseer (2018) witnessed that traits namely peduncle length, spike length and biological yield plot $^{-1}$ showed positive and significant associations with grain yield under moderate salinity. These kinds of results were also reported by Goudarzi and Pakniyat (2008) and Chamekh et al. (2017). The current study also exhibited significant $(P<0.05)$ and positive correlations among various traits under salinity stress conditions, such days to $75 \%$ heading with plant height; $\left(r=0.64^{* *}\right)$, plant height with grains spike ${ }^{-1}\left(r=0.35^{* *}\right)$, tillers plant ${ }^{-1}$ with spikelets spike ${ }^{-1}\left(r=0.32^{* *}\right)$, relative water content $\left(r=0.34^{*}\right)$ and harvest index $\left(r=0.38^{* *}\right)$, spike length with spikelets spike ${ }^{-1}\left(\mathrm{r}=0.34^{* *}\right)$, grains spike ${ }^{-1}$ with biological yield plot $^{-1}$ $\left(r=0.37^{* *}\right)$ and relative water content $\left(r=0.26^{*}\right)$, spikelets spike ${ }^{-1}$ with grains spike ${ }^{-1} \quad\left(r=0.30^{*}\right)$, grains spike ${ }^{-1}$ with seed index $\left(r=0.47^{* *}\right)$, seed index with biological yield plot $^{-1} \quad\left(r=0.31^{*}\right)$, biological yield plot $^{-1}$ with leaf area $\left(r=0.47^{* *}\right)$, relative water content with $\mathrm{K}^{+}\left(\mathrm{r}=0.29^{*}\right)$ and leaf area with $\mathrm{K}^{+}\left(\mathrm{r}=0.24^{*}\right)$. Overall, an intensive selection for these growth, yield and physiological traits will ultimately improve seed yield in wheat under salinity stress conditions. Dashti et al. (2012) observed significantly negative associations between $\mathrm{K}^{+} / \mathrm{Na}^{+}$ratio and $\mathrm{Na}^{+}$concentration, while $\mathrm{K}^{+} / \mathrm{Na}^{+}$ratio was significantly and positively interrelated with $\mathrm{K}^{+}$. Asgari et al. (2012) also reported similar associations under salinity, including tillers plant ${ }^{1}$ with spikelets spike ${ }^{-1}$, grains spike ${ }^{-1}, \mathrm{~K}^{+}$and $\mathrm{K}^{+} / \mathrm{Na}^{+}$ratio, spike length with spikelets spike ${ }^{-1}$, $\mathrm{K}^{+}$with $\mathrm{K}^{+} / \mathrm{Na}^{+}$ratio and seed index with tillers plant $^{-1}$, spike length and spikelets spike ${ }^{-1}$.

Table 1. Mean squares of various traits of bread wheat genotypes grown in non-saline and salinity stress conditions

\begin{tabular}{|c|c|c|c|c|c|}
\hline Source of variance & $\begin{array}{l}\text { Replication } \\
\text { D.F. (2) }\end{array}$ & $\begin{array}{l}\text { Genotypes (G) } \\
\text { D.F. (21) }\end{array}$ & $\begin{array}{l}\text { Treatments (T) } \\
\text { D.F. (1) }\end{array}$ & $\begin{array}{l}\text { G x T interaction } \\
\text { D.F. (21) }\end{array}$ & $\begin{array}{l}\text { Error } \\
\text { D.F. (82) }\end{array}$ \\
\hline Days to $75 \%$ heading & 0.11 & 14.83 & 1781.16 & 5.84 & 1.65 \\
\hline Days to $75 \%$ maturity & 3.47 & $13.82^{m}$ & 3229.66 & 11.51 & 1.70 \\
\hline Plant height & 26.8 & 325.2 & 14247.4 & 32.2 & 23.2 \\
\hline Tillers plant $^{-1}$ & 0.656 & $18.043^{* *}$ & $119.603^{* *}$ & $0.391^{\mathrm{ns}}$ & 0.652 \\
\hline Spike length & 1.1204 & $6.4196^{* *}$ & $68.4254^{* *}$ & $1.3863^{*}$ & 0.5564 \\
\hline Spikelets spike $^{-1}$ & 0.387 & 17.301 & $612.43^{\prime \prime}$ & $1.081^{\text {ns }}$ & 0.926 \\
\hline Grains spike $^{-1}$ & 5.20 & 471.59 & 4956.18 & $12.08^{\mathrm{ns}}$ & 6.45 \\
\hline Grain yield plot ${ }^{-1}$ & 0.00023 & $0.17128^{* *}$ & $3.17478^{* *}$ & $0.00357^{\mathrm{ns}}$ & 0.00449 \\
\hline Biological yield plot $^{-1}$ & 0.00692 & 0.12351 " & 4.76178 & 0.03918 & 0.00833 \\
\hline Harvest index & 0.96 & 127.07 & 1464.24 & 5.80 & 1.98 \\
\hline Seed index & 23.10 & 80.14 & 2796.45 & $4.80^{\text {nS }}$ & 6.20 \\
\hline Leaf area & 0.271 & 49.420 & 904.909 & 4.429 & 1.198 \\
\hline Relative water content & 123.8 & $233.1^{\prime \prime}$ & 51280.2 & 249.2 & 68.8 \\
\hline Chlorophyll & 0.53 & 198.46 & 3382.85 & $6.08^{\text {ns }}$ & 5.55 \\
\hline $\mathrm{K}^{+}$ & 0.0977 & 0.4424 & 22.5724 & 0.1106 & 0.0538 \\
\hline $\mathrm{Na}^{+}$ & 0.00963 & 0.16203 & $2.98841^{\prime \prime}$ & 0.02383 & 0.00847 \\
\hline $\mathrm{K}^{+} / \mathrm{Na}^{+}$ratio & 0.111 & 7.526 & 167.818 & 0.562 & 0.364 \\
\hline
\end{tabular}

", represent significant at 1 and $5 \%$ of probability level, respectively, while ns shows non-significant 
Pak. J. Agri., Agril. Engg., Vet. Sci., 2020, 36 (2)

Table 2. Correlation coefficient (r) between morphological, physiological and yield traits of bread wheat genotypes in non-saline conditions

\begin{tabular}{|c|c|c|c|c|c|c|c|c|c|c|c|c|c|c|c|}
\hline Traits & DM & DH & PH & TP & SL & SPS & GS & GYP & SI & BP & HI & CHL & RWC & LA & $\mathbf{N}$ \\
\hline $\mathrm{DH}$ & $-0.21^{n s}$ & & & & & & & & & & & & & & \\
\hline $\mathrm{PH}$ & $-0.20^{\text {ns }}$ & $-0.11^{\mathrm{ns}}$ & - & & & & & & & & & & & & \\
\hline TP & $0.15^{\mathrm{ns}}$ & $0.41^{\prime}$ & $0.04^{\mathrm{ns}}$ & - & & & & & & & & & & & \\
\hline SL & $0.12^{\text {ns }}$ & 0.32 & $-0.12^{\text {ns }}$ & 0.42 & - & & & & & & & & & & \\
\hline SPS & $0.18^{\text {ns }}$ & 0.51 & $-0.19^{n s}$ & 0.31 & 0.31 & - & & & & & & & & & \\
\hline GS & $0.09^{\text {ns }}$ & 0.34 & $0.10^{\text {ns }}$ & 0.26 & 0.49 & 0.33 & - & & & & & & & & \\
\hline GYP & 0.25 & 0.53 & $0.06^{\text {ns }}$ & 0.63 & 0.59 & 0.49 & $0.40^{\circ}$ & - & & & & & & & \\
\hline SI & $0.20^{\mathrm{ns}}$ & 0.26 & -0.67 & $0.09^{n s}$ & 0.35 & 0.30 & $0.18^{\mathrm{ns}}$ & 0.29 & - & & & & & & \\
\hline BYP & $0.19^{n s}$ & $0.16^{\text {ns }}$ & 0.29 & $0.41^{\prime \prime}$ & 0.37 & $0.17^{\text {ns }}$ & $0.12^{\mathrm{ns}}$ & 0.56 & $0.01^{\text {ns }}$ & - & & & & & \\
\hline $\mathrm{HI}$ & $0.6^{\mathrm{ns}}$ & 0.27 & -0.33 & $0.13^{\text {ns }}$ & 0.32 & $0.20^{\text {ns }}$ & 0.25 & 0.24 & 0.32 & 0.58 & - & & & & \\
\hline $\mathrm{CHL}$ & $0.21^{\text {ns }}$ & $0.17^{\text {ns }}$ & -0.28 & 0.33 & 0.33 & 0.31 & $0.16^{\text {ns }}$ & $0.17^{\text {ns }}$ & $0.49^{\prime \prime}$ & $0.04^{\text {ns }}$ & $0.16^{\mathrm{ns}}$ & - & & & \\
\hline RWC & $0.20^{\text {ns }}$ & 0.45 & $-0.05^{\text {ns }}$ & 0.59 & 0.42 & 0.48 & 0.53 & 0.53 & 0.35 & 0.30 & 0.23 & 0.48 & - & & \\
\hline LA & $0.21^{\text {ns }}$ & $-0.02^{\text {ns }}$ & 0.36 & $0.15^{\mathrm{ns}}$ & $0.32^{*}$ & 0.12 & $0.11^{\mathrm{ns}}$ & $0.16^{\text {ns }}$ & $0.35^{* \prime \prime}$ & $0.12^{\mathrm{ns}}$ & $0.01^{\mathrm{ns}}$ & $0.17^{\mathrm{ns}}$ & $0.16^{\mathrm{ns}}$ & & \\
\hline $\mathrm{N}$ & $-0.07^{n s}$ & $0.15^{\mathrm{ns}}$ & -0.24 & $0.02^{\text {ns }}$ & $-0.03^{\text {ns }}$ & $-0.05^{\text {ns }}$ & $-0.01^{\mathrm{ns}}$ & $0.01^{\mathrm{ns}}$ & $0.16^{\text {ns }}$ & $0.14^{\text {ns }}$ & $0.20^{\text {ns }}$ & $0.01^{\mathrm{ns}}$ & $-0.04^{\text {ns }}$ & $0.18^{\text {ns }}$ & - \\
\hline $\mathrm{K}$ & $0.40^{\circ}$ & $0.40^{\circ}$ & -0.35 & $0.20^{n s}$ & 0.28 & 0.48 & $0.12^{\mathrm{ns}}$ & 0.28 & 0.28 & $0.04^{\mathrm{ns}}$ & $0.15^{\mathrm{ns}}$ & $0.20^{\mathrm{ns}}$ & $0.21^{\mathrm{ns}}$ & $0.21^{\mathrm{ns}}$ & $0.09^{\text {ns }}$ \\
\hline KNR & $0.22^{\text {ns }}$ & $0.01^{n s}$ & $0.06^{\mathrm{nS}}$ & $0.06^{\text {ns }}$ & $0.20^{\text {ns }}$ & 0.27 & $0.11^{\mathrm{ns}}$ & $0.13^{\text {ns }}$ & $-0.03^{n s}$ & $0.15^{\text {ns }}$ & $-0.10^{\text {ns }}$ & $0.12^{n s}$ & $0.13^{\text {ns }}$ & 0.34 & 0.82 \\
\hline
\end{tabular}

$\mathrm{DH}=$ Days to $75 \%$ heading; $\mathrm{DM}=$ Days to $75 \%$ maturity; $\mathrm{PH}=\mathrm{Plant}$ height; TP= Tillers plant ${ }^{-1} ; \mathrm{SL}=\mathrm{Spike}$ length; $\mathrm{SPS}=\mathrm{Spikelets}$ spike ${ }^{-1} ; \mathrm{GS}=\mathrm{Grains}$ spike ${ }^{-1}$; GYP= Grain yield plot $^{-1} ; \mathrm{Sl}=\mathrm{Seed}$ index; $\mathrm{BP}=$ Biological yield plot $^{-1} ; \mathrm{HI}=$ Harvest index; $\mathrm{CHL}=$ Chlorophyll; RWC= Relative water content; $\mathrm{LA}=\mathrm{Leaf}$ area; $\mathrm{N}=\mathrm{N}^{+} ; \mathrm{K}=\mathrm{K}^{+} ; \mathrm{KNR}=\mathrm{K}^{+} / \mathrm{N}^{+}$ratio

Table 3. Correlation coefficient $(r)$ between morphological, physiological and yield traits of bread wheat genotypes under salinity stress conditions

\begin{tabular}{|c|c|c|c|c|c|c|c|c|c|c|c|c|c|c|c|c|}
\hline Traits & DM & DH & $\mathrm{PH}$ & TP & $\mathrm{SL}$ & SPS & GS & GYP & SI & BP & \begin{tabular}{|l}
$\mathrm{HI}$ \\
\end{tabular} & $\mathrm{CHL}$ & RWC & LA & $\mathbf{N}$ & $\mathrm{K}$ \\
\hline $\mathrm{DH}$ & \begin{tabular}{|l|l|}
$-0.17^{\mathrm{ns}}$ \\
\end{tabular} & - & & & & & & & & & & & & & & \\
\hline $\mathrm{PH}$ & $0.16^{\mathrm{ns}}$ & 0.64 & - & & & & & & & & & & & & & \\
\hline TP & $0.22^{\mathrm{ns}}$ & -0.39 & \begin{tabular}{|l|}
-0.41 \\
\end{tabular} & - & & & & & & & & & & & & \\
\hline $\mathrm{SL}$ & $\begin{array}{l}-0.06^{\mathrm{ns}} \\
\end{array}$ & -0.44 & -0.45 & $0.20^{\text {ns }}$ & - & & & & & & & & & & & \\
\hline SPS & $-0.09^{\text {ns }}$ & -0.42 & -0.25 & 0.32 & 0.34 & - & & & & & & & & & & \\
\hline GS & $0.09^{\text {ns }}$ & -0.44 & $0.35^{\prime}$ & $0.06^{\mathrm{ns}}$ & 0.55 & 0.30 & - & & & & & & & & & \\
\hline GYP & $-0.04^{\mathrm{ns}}$ & -0.36 & \begin{tabular}{|l|}
-0.33 \\
\end{tabular} & $0.24^{\text {ns }}$ & 0.37 & 0.46 & 0.43 & - & & & & & & & & \\
\hline SI & $0.03^{\text {ns }}$ & -0.51 & \begin{tabular}{|l|}
-0.32 \\
\end{tabular} & 0.31 & $0.16^{\text {ns }}$ & $0.14^{\mathrm{ns}}$ & 0.47 & 0.52 & - & & & & & & & \\
\hline BYP & $-0.16^{\mathrm{ns}}$ & $-0.05^{\mathrm{ns}}$ & $-0.09^{\text {ns }}$ & $-0.08^{\text {ns }}$ & 0.24 & $0.30^{\circ}$ & $0.37^{\circ}$ & 0.70 & 0.31 & - & & & & & & \\
\hline $\mathrm{HI}$ & $0.18^{\text {ns }}$ & -0.41 & \begin{tabular}{|l|}
-0.29 \\
\end{tabular} & 0.38 & $0.15^{\text {ns }}$ & $0.11^{\mathrm{ns}}$ & $0.13^{\text {ns }}$ & 0.26 & 0.26 & $\mid-0.41$ & & & & & & \\
\hline $\mathrm{CHL}$ & $0.08^{\text {ns }}$ & $-0.28^{-}$ & $\mid-0.24$ & $0.18^{\text {ns }}$ & $0.13^{\text {ns }}$ & $0.07^{\mathrm{ns}}$ & $0.07^{\text {ns }}$ & $0.17^{\mathrm{ns}}$ & $0.03^{\text {ns }}$ & $0.00^{\text {ns }}$ & $0.23^{\text {ns }}$ & - & & & & \\
\hline RWC & $0.02^{\text {ns }}$ & -0.50 & \begin{tabular}{|l|}
-0.40 \\
\end{tabular} & 0.34 & 0.32 & $0.21^{\mathrm{ns}}$ & 0.26 & 0.50 & $0.51^{\prime}$ & $0.20^{\text {ns }}$ & $0.23^{\text {ns }}$ & $0.13^{\text {ns }}$ & & & & \\
\hline LA & $0.08^{\text {ns }}$ & -0.28 & $-0.06^{\text {ns }}$ & $0.20^{\text {ns }}$ & $0.22^{\text {ns }}$ & -0.36 & $0.23^{\text {ns }}$ & 0.45 & 0.47 & 0.47 & $0.03^{\text {ns }}$ & $0.13^{\text {ns }}$ & $0.23^{\text {ns }}$ & - & & \\
\hline $\mathrm{N}$ & $0.01^{\mathrm{ns}}$ & $0.20^{n s}$ & \begin{tabular}{|l|l}
$0.01^{\mathrm{nS}}$ \\
\end{tabular} & $0.01^{n s}$ & $0.01^{\mathrm{ns}}$ & $0.02^{\text {ns }}$ & $\begin{array}{l}-0.04^{n \mathrm{~s}} \\
\end{array}$ & $0.10^{\text {ns }}$ & $0.01^{\mathrm{nS}}$ & $0.21^{\text {ns }}$ & $0.17^{n \mathrm{~s}}$ & $-0.11^{\mathrm{ns}}$ & \begin{tabular}{|l|l}
$0.17^{\mathrm{nS}}$ \\
\end{tabular} & $0.17^{n \mathrm{SS}}$ & - & \\
\hline $\mathrm{K}$ & $0.02^{\text {ns }}$ & -0.46 & -0.32 & 0.25 & $0.23^{\text {ns }}$ & 0.30 & 0.34 & 0.32 & -0.29 & $0.16^{\text {ns }}$ & $0.23^{\text {ns }}$ & $0.04^{\mathrm{ns}}$ & 0.29 & 0.24 & $0.09^{\text {ns }}$ & \\
\hline KNR & $-0.04^{\mathrm{ns}}$ & -0.35 & \begin{tabular}{|l}
$-0.22^{n s}$ \\
\end{tabular} & $0.07^{\text {ns }}$ & $0.09^{n s}$ & $0.07^{n s}$ & $0.15^{\mathrm{ns}}$ & $-0.01^{n s}$ & $0.07^{n s}$ & $-0.14^{\mathrm{ns}}$ & $0.21^{\mathrm{ns}}$ & $0.06^{\mathrm{ns}}$ & $-0.04^{\mathrm{ns}}$ & $-0.11^{\mathrm{ns}}$ & $-0.91^{\prime \prime}$ & $0.23^{\text {ns }}$ \\
\hline
\end{tabular}

\section{CONCLUSION}

Correlation results in non-saline and saline soil exhibited that a number of traits made positively significant interrelationships with grain yield plot $^{-1}$, such as spike length, spikelets spike ${ }^{-1}$, grains spike ${ }^{-1}$, seed index, biological yield plot $^{-1}$, relative water content and $\mathrm{K}^{+}$. Hence, these traits may be used as selection criteria to develop high yielding wheat genotypes for nonsaline and salinity stress conditions.

\section{ACKNOWLEDGEMENTS}

The authors wish to acknowledge to the Director, Nuclear Institute of Agriculture, Tandojam for providing facilities in the experimental field and laboratory to carry out the present research work.

\section{AUTHOR'S CONTRIBUTION}

N. Gandahi: Conducted the experiments and wrote the manuscript

A. W. Baloch: Design the experiment and corrected the final version of manuscript

S. A. Sarki: Helped in revision

M. M. Lund: Helped in revision
M. N. Kandhro: Technical evaluation of manuscript

\section{REFERENCES}

Ahmad, M., A. Shahzad, M. Asif and M. Sajad. 2013. Genetic analysis of yield and yield contributing quantitative traits in bread wheat under sodium chloride salinity. Journal of Agricultural Sciences, 5 (6): 156-163.

Aman, J., K. Bantte, S. Alamerew and D. B. Sbhatu. 2020. Correlation and path coefficient analysis of yield and yield components of quality protein maize (Zea mays L.) hybrids at Jimma, Western Ethiopia. International Journal of Agronomy, 2020: 9651537.

Ansari, R. and T. J. Flowers. 1986. Leaf to leaf distribution of ions in some monocotyledonous plants grown under saline conditions. In: Prospects for Biosaline Research, pp.167-181.

Asgari, H. R., W. Cornelisb and P. V. Dammec. 2012. Salt stress effect on wheat (Triticum aestivum L.) growth and leaf ion concentrations. International Journal of Plant Production, 6 (2): 195-208. 
Baloch, A. W., S. M. Bhatti, M. Baloch, Q. D. Jogi and M. N. Kandhro. 2016. Correlation analysis of various metric traits with grain yield and heritability estimation in rice genotypes. Pakistan Journal of Agriculture, Agriculture Engineering and Veterinary Sciences, 32 (2): 136-142.

Baloch, A. W., M. Baloch, I. A. Baloch, S. N. Mari, D. K. Mandan and S. A. Abro. 2014. Association and path analysis in advance Pakistani bread wheat genotypes. Pure and Applied Biology, 3 (3): 115-120.

Baloch, M., M. H. Kaleri, A. W. Baloch, T. A. Baloch, N. Gandahi, Q. Jogi, L. A. Bhutto and J. A. Hakro. 2016. Phenotypic correlation and heritability analysis in sunflower (Helianthus annuus L.) germplasm. Pure and Applied Biology, 5 (3): 641-646.

Banglian, H., Y. Yang, T. Luo, S. Wu, X. Du, D. Cai, E.N. Loo and B. Huang. 2017. Correlation, regression and path analyses of seed yield components in Crambe abyssinica, a promising industrial oil crop. Journal of Plant Breeding and Genetics, 59 (2): 215-221.

Belderok, B. 2000. Bread-making Quality of Wheat: A Century of Breeding in Europe. Kluwer Academic Publishers, Netherlands.

Bonnet, M., O. Camares and P. Veisseire. 2000. Effect of zinc and influence of Acremonium lolii on growth parameters, chlorophyll a fluorescence and antioxidant enzyme activities of ryegrass (Lolium perenne L. CV Apollo). Journal of Experimental Botany, 51 (346): 945-953.

Chamekh, Z., C. Karmous, S. Ayadi, A. Sahli, M. B. Fraj, S. Yousfi, S. Rezgui, N. B. Aissa, M. D. Serret, I. McCann, Y. Trifa, H. Amara and J. L. Araus. 2017. Comparative performance of $\delta^{13} \mathrm{C}$, ion accumulation and agronomic parameters for phenotyping durum wheat genotypes under various irrigation water salinities. Annals of Applied Biology, 170 (2): 229-239.

Chavan, B. R., L. N. Jawale and A. V. Shinde. 2020. Correlation and path analysis studies in finger millet for yield and yield contributing traits (Eleusine coracana L. Gaertn). International Journal of Chemical Studies, 8 (1): 2911-2914.

Chitralekha, P. K., N. K. Chandrakar, U. Banjare and M. Densena. 2018. Estimation of correlation coefficient study of some quantitative traits in wheat. Annals of Plant Sciences, 7 (2): 2078-2081.
Dashti, H., M. R. Bihamta, H. Shirani and M. M. Majidi. 2012. Genetic analysis of salt tolerance in vegetative stage in wheat (Triticum aestivum). Plant Omics, 5 (1): 19-23.

Dhami, N. B., M. Kandel, S. B. Gurung and J. Shrestha. 2018. Agronomic performance and correlation analysis of finger millet genotypes (Elusine coracana L.). Malaysian Journal of Sustainable Agriculture, 2 (2): 16-18.

Gadalla, M. A., S. I. Milad, M. Y. Mabrook, Y. A. Amira and M. A. Gouda. 2017. Evaluation of some Egyptian bread wheat (Triticum aestivum) cultivars under salinity stress. Alexandra Science Exchange Journal, 38 (2): 261-270.

Garciadeblás, B., M. E. Senn, M. A. Bañuelos and A. Rodriquez-Navarro. 2003. Sodium transport and HKT transporters: the rice model. The Plant Journal, 34 (6): 788-801.

Carillo, P., M. G. Annunziata, G. Pontecorvo, A. Fuggi and $P$. Woodrow. 2011. Salinity stress and salt tolerance," in Abiotic Stress in Plants-Mechanisms and Adaptations, A. K. Shanker and B. Venkateswarlu, Eds., pp. 21-38.

GoP. 2019. Economic Survey of Pakistan. 2019. Ministry of Food and Agriculture Government of Pakistan, Economic Advisory Wing, Finance Division, Islamabad.

Gorgieva, B., K. Ilija, M. Sasa, M. Ruzdik, K. Emilija and K. Biljana. 2015. Correlation and path analysis in sunflower (Helianthus annuus L.). Hellia, 38 (63): 201-2010.

Goudarzi, M. and H. Pakniyat. 2008. Evaluation of wheat cultivars under salinity stress based on some agronomic and physiological traits. Journal of Agriculture and Social Sciences, 4 (3): 35-38.

Hailu, A., S. Alamerew, M. Nigussie and E. Assefa. 2016. Correlation and path coefficient analysis of yield and yield associated traits in barley (Hordeum vulgare L.) germplasm. Advance Crop Science and Technology, 4 (2): 216.

Hasan, M. J., M. U. Kulsum, A. Akter, A. S. M. Masuduzzaman and M. S. Ramesha. 2013. Genetic variability and character association for agronomic traits in hybrid rice (Oryza Sativa L.). Bangladesh Journal of Plant Breeding and Genetics, 24 (1): 45-51.

Kandel, M., S. K. Ghimire, B. R. Ojha and J. Shrestha. 2018. Genetic diversity for heat tolerant related traits in maize inbred lines. Agricultura, 105 (1-2): 23-34. 
Khan, A. 2020. Performance of different bread wheat varieties for yield and yield attributes under diallel combinations. Annals of Agriculture Science, 14 (2): 25-34.

Kharel, L., S. K. Ghimire, J. Shrestha, C. B. Kunwar and S. Sharma. 2018. Evaluation of rice genotypes for its response to added fertility levels and induced drought tolerance during reproductive phase. Journal of Agriculture Research, 5 (1): 13-18.

Kumar, A., P. Bandhu and A. Das. 2005. Salt tolerance and salinity effects on plants: a review. Ecotoxicology and Environmental Safety, 60 (3): 324-349.

Lichtenthaler, H. K. 1987. Chlorophylls and carotenoids: Pigments of photosynthetic biomembranes. Methods in Enzymology, 148: 350-382.

Marcussen, T., S. R. Sandve, L. Heier, M. Spannagl, M. Pfeifer, K. S. Jakobsen, B. B. Wulff, B. Steuernagel, K. F. Mayer, O. A. Olsen. 2014. Ancient hybridization among the ancestral genomes of bread wheat. Science, 345 (6194): 125-129.

Mengesha, G. H., F. M. Hailemariam, T. T. Mindaye, B. Lakew and R. P. S. Verma. 2019. Correlation and path analysis of yield, yield contributing and malt quality traits of Ethiopian sorghum (Sorghum bicolor (L.) Moench) genotypes. African Journal of Plant Science, 13 (8): 209-220.

Munns, R. and M. Gilliham. 2015. Salinity tolerance of crops-what is the cost? New Phytologist, 208 (3): 668-673.

Naggar, A. M. M., S. R. S. Sabry, M. M. M. Atta and O. M. A. Aleem. 2015. Field screening of wheat (Triticum aestivum L.) genotypes for salinity tolerance at three locations in Egypt. Journal of Agriculture and Ecology Research International, 4 (3): 88-104.

Parihar, R., A. P. Agrawal, M. Burman and M. G. Minz. 2018. Relationship between grain yield and other yield attributing characters in wheat under terminal heat stress. Journal of Pharmacology and Phytology, 7 (1): 21142117.

Qados, A. M. A. 2011. Effect of salt stress on plant growth and metabolism of bean plant Vicia faba (L.). Journal of the Saudi Society of Agricultural Sciences, 10 (1): 7-15.

Rattar, F. 2019. Inter-relationship analysis of quantitative traits in bread wheat genotypes. M.Sc. Thesis submitted through department of Plant Breeding and Genetics to Sindh Agriculture University, Tandojam.
Rout, S., S. A. Kerkhi and C. Charupriya. 2018. Character association and path analysis among yield components in Indian Rapeseed [Brassica napus (L.) Czern and Coss]. International Journal of Current Microbiology and Applied Sciences, 7 (1): 50-55.

Sairam, R. K., K. V. Rao and G. C. Srivastava. (2002). Differential response of wheat genotypes to long term salinity stress in relation to oxidative stress, antioxidant activity and osmolyte concentration. Plant Science, 163 (5): 1037-1046.

Shah, T., A. Z. Khan, M. Numan, W. Ahmad, M. Zahoor, M. Ullah and A. Jalal. 2017. Nutrient uptake and yield of wheat varieties as influenced by foliar potassium under drought condition. Cercetari Agronomice in Moldova, 50 (2): 5-20.

Singh, O., S. K. Dwivedi, R. S. Shukla, R. K. Samaiya, A. Upadhayay, D. Vasht, M. Singh and S. Prajapati. 2017. Correlation studies in diverse lines of wheat (Triticum aestivum L.) under restricted irrigation, International Journal of Pure and Applied Bioscience, 5 (1): 379-388.

Sohail, A., H. Rehman, Farhatullah, S. M. A. Shah, T. Burni and S. Ali. 2018. Evaluation of $\mathrm{F}_{4}$ bread wheat (Triticum aestivum L.) genotypes for genetic variability, heritability, genetic advance and correlation studies. Journal of Plant Breeding and Genetics, 6 (1): 1-7.

Solanki, R. S., K. Pawan, S. P. Mishra and S. R. Ramgiry. 2017. Contribution of agromorphological traits in seed yield of Indian rapeseed (Brassica napus L. Czern and Coss) germplasm under rainfed condition. International Journal of Current Microbiology and Applied Sciences, 6 (9): 2281-2286.

Surje, D. T. and D. K. De. 2014. Correlation coefficient study in oat (Avena sativa L.) genotypes for fodder and grain yield characters. Journal of Agriculture and Technology, 1 (1): 89-93.

Wang, M. and G. Xia. 2018. The landscape of molecular mechanisms for salinity tolerance in wheat. The Crop Journal, 6 (1): 42-47.

Waseer, M. W. 2018. Evaluation of bread wheat (Triticum aestivum L.) genotypes for higher grain yield under salt stress. M.Sc. Thesis submitted through department of Plant Breeding and Genetics to Sindh Agriculture University, Tandojam.

(Received: June 29, 2020; Accepted: December 22, 2020) 